\title{
CHOROIDAL SARCOMA WITH METASTASIS IN THE OPPOSITE ORBIT*
}

BY

J. FOSTER, W. HENDERSON, J. W. COWIE AND D. S. F. HARRIMAN

United Leeds Hospitals

THE DETAILS of this case are submitted for publication partly because of its rarity, only one similar case having been found in the literature (Philps and Ashton, 1949), and partly because of certain interesting details in the diagnosis and treatment. The patient described by Philps and Ashton, a woman aged 59 years, developed 2D hypermetropia, exophthalmos, and a palpable tumour in the left orbit 10 years after excision of the right eye for a choroidal melanoma. A tumour was removed from the muscle cone by a lateral orbitotomy, and 6 months later the loss of central vision, hypermetropia, and exophthalmos had been cured. Comparison of the sections of the orbital tumour with that of the choroid left little doubt that the second tumour was a metastasis from the first. Our own case is strikingly similar.

\section{Case Report}

A woman aged 56 had her right eye excised in 1951 (by Mr. F. Gamm in the Huddersfield Infirmary) for melanoma of the choroid; 4 years later, in October, 1955, the vision in the left eye began to fail rapidly with simultaneous development of exophthalmos, and she was referred by Mr. Gamm to the Leeds Infirmary for a contrast-medium $x$-ray of the orbit by the technique of Groves and Cowie (1955).

Examination.-In December, 1955, she had $4 \mathrm{~mm}$. of exophthalmos (Fig. 1), papilloedema, a hemianopic scotoma to the outer side of fixation (to white objects of $3 / 2000$

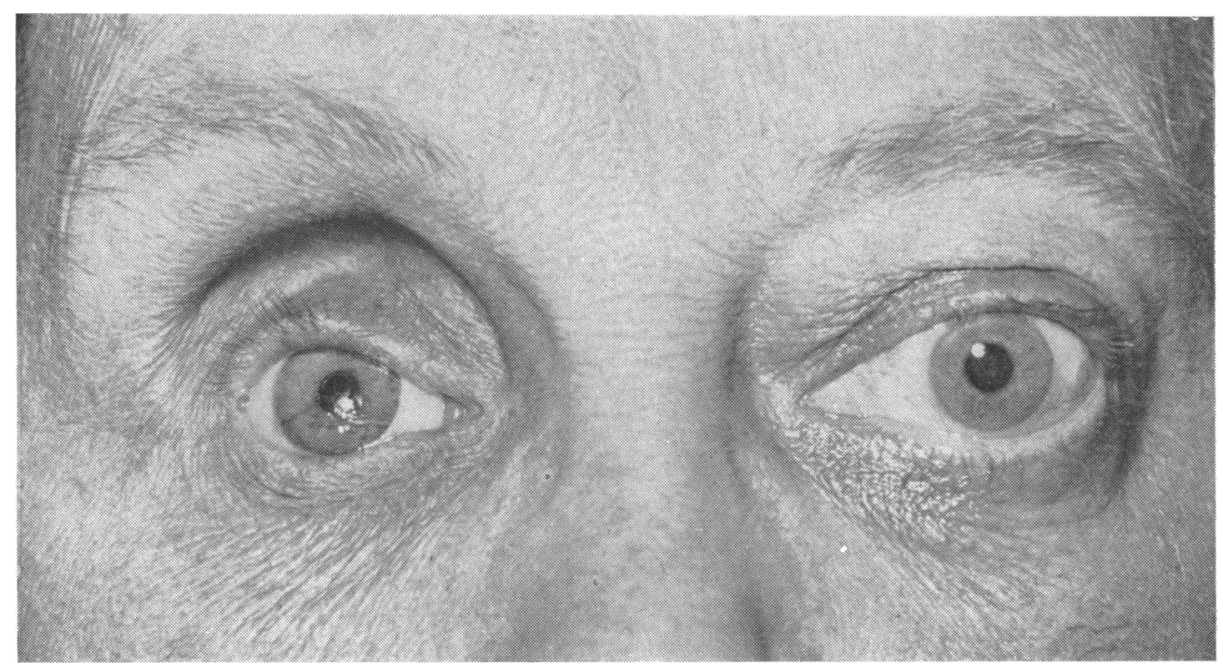

FIG. 1.-4-mm. left exophthalmos measured by Mutch's proptometer. 
and 15/2000), and marked limitation of eye movement up and in (Fig. 2).

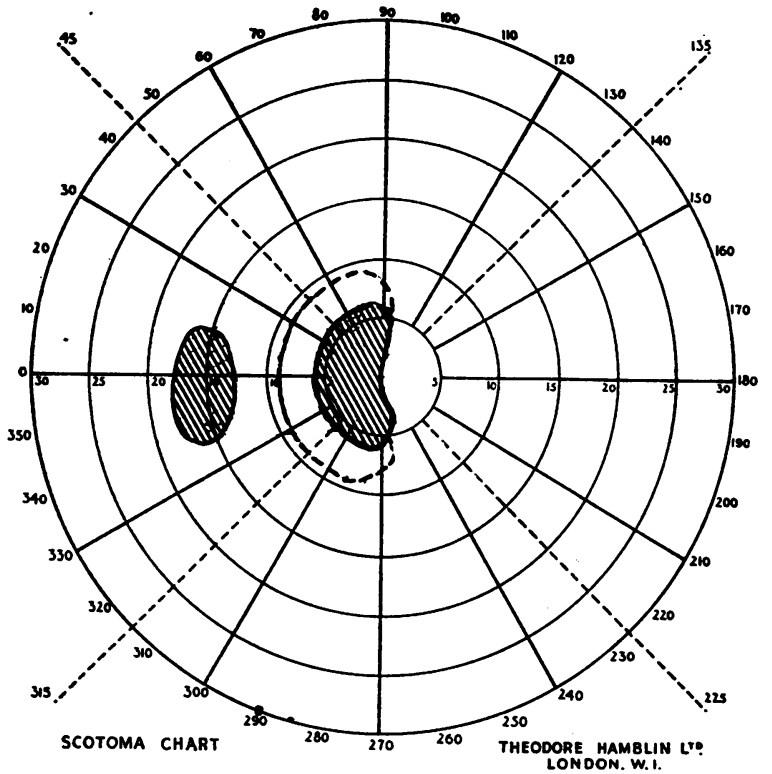

FIG. 2.-Scotomata 3/2000 and $15 / 2000$ left eye, on December 15, 1955.

The visual acuity with $+2 \mathrm{D}$ sph./ $+0 \cdot 25 \mathrm{D}$ cyl., $100^{\circ}$ was $6 / 24$, add $+2 \cdot 25=$ N.10. A week later the scotoma had extended (Fig. 3) and the refraction with $+3 \cdot 5 \mathrm{D}$ cyl. was $6 / 36$.

Fig. 3.-Scotomata $15 / 2000$ left eye, on December 22 , 1955.

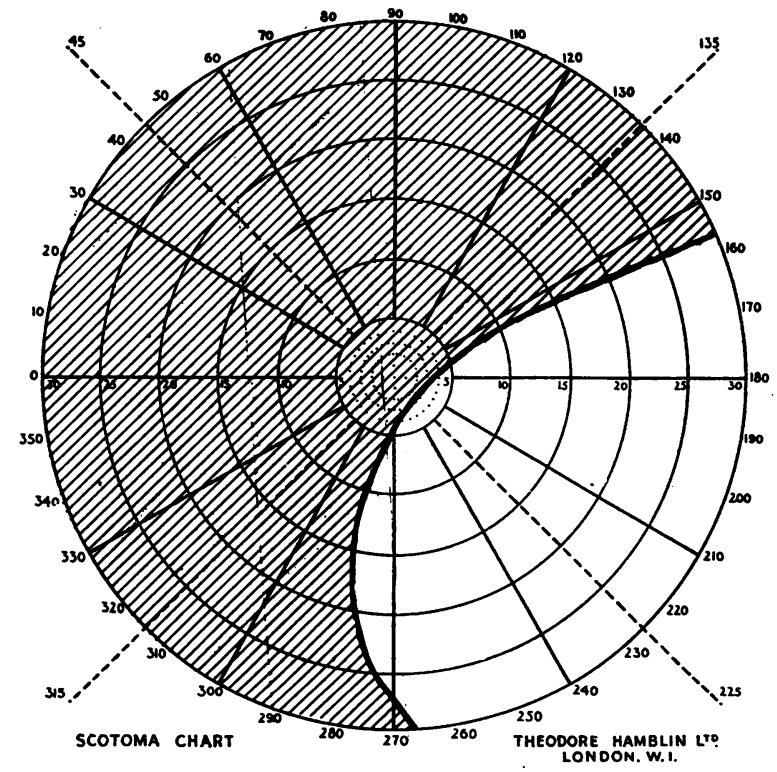

Diodone X-Ray (J. W. Cowie). $-3.5 \mathrm{ml} .17 \cdot 5$ per cent. Diodone in 2 per cent. Novocain was injected into the muscle cone and routine films were taken.

The appearances were rather unusual, as there was no true clover shape representing the muscle cone in the antero-posterior view, but a hollow, slightly irregular, circular opacity (Fig. 4, overleaf).

On the lateral view a "shelf" was discerned medium above and below the site of a normal muscle cone (Fig. 5, overleaf).

The appearances were those of a large mass in the muscle cone and mainly, if not entirely, confined by it. 


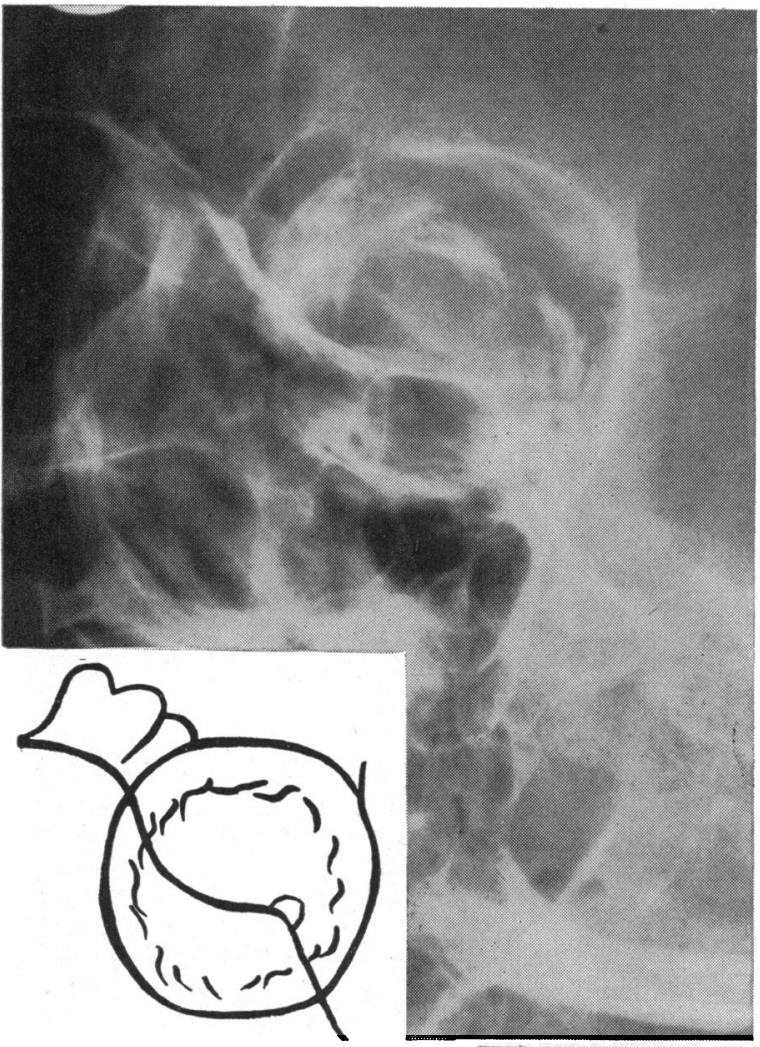

Fig. 4.-Diodone $x$-ray, antero-posterior view, showing a hollow, slightly irregular, circular opacity.

FIG.5.-Diodone $x$-ray, lateral view, showing a shelf medium above and below the normal muscle cone.

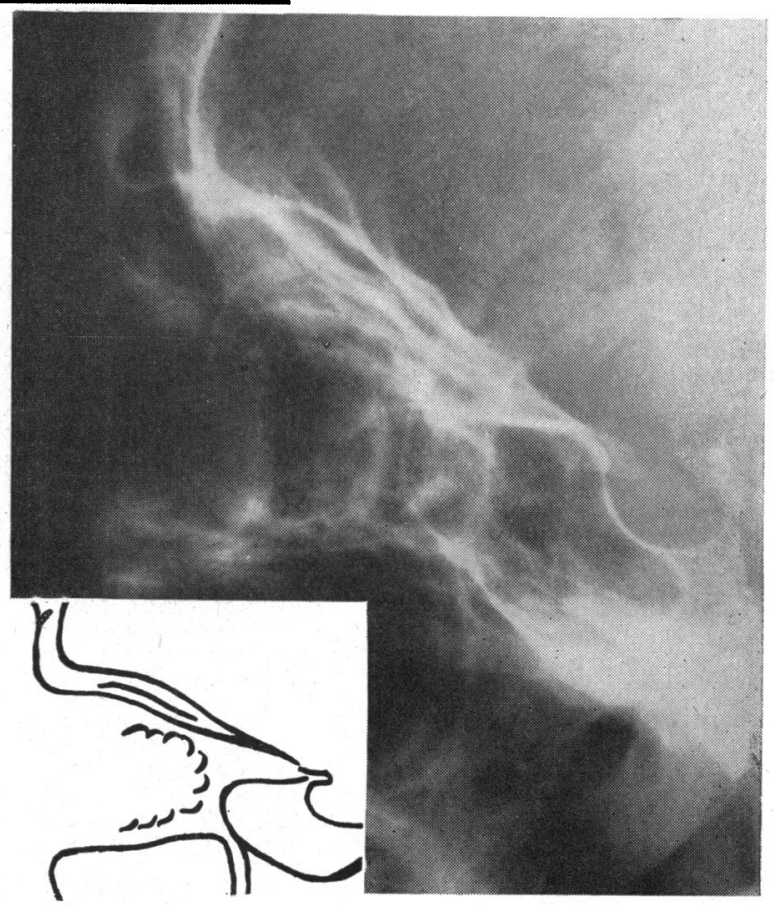


Diagnosis.-At this time it was thought that there might be a metastasis from the excised eye in the orbit, or a growth arising from the left optic nerve sheath. As the hemianopic scotoma was located to the outer side of fixation, it was felt that the growth was probably located on the inner side of the nerve and that a transfrontal exploration was indicated.

The patient was referred to Mr. W. R. Henderson, who expressed the opinion that an exploration would only be justified if the patient were willing to run the risk of damage to the optic nerve during the operation, which would produce immediate total blindness. Since loss of the vision and ultimate loss of the eye would certainly occur in time, and the patient had already developed another mm. of exophthalmos, consent was given.

Operation (Mr. W. R. Henderson).-This was performed on February 6, 1956. A left transfrontal bone flap was made. The dura was stripped back to the sphenoidal ridge, and the orbital roof was removed from the frontal sinus back to the sphenoidal fissure. Retraction of the superior muscles laterally and of the trochlear nerve and muscles medially revealed a rounded tumour medial to the optic nerve which was displaced laterally but not invaded by the tumour. At first sight the tumour looked like a very ripe cherry but it was later found to be considerably larger, the size of a walnut, as it extended down to the orbital floor. It had a thin smooth capsule which at first dissected easily from orbital structures but deeper down there was no line of separation. The capsule was opened and a dark red, soft, solid tumour containing small blackish areas was scooped out. As the remaining capsule was firmly adherent and could not be removed without damaging normal structures the operation was terminated.

Pathology (Dr. D. S. F. Harriman, Leeds Infirmary Neuro-surgical Department).After fixation in Bouin's fluid, the tissues were embedded in paraffin and stained by haematoxylin and eosin, Holme's silver method, and Fontana's method for melanin granules.

The tumour -proper consisted mainly of fairly closely packed plump spindle cells arranged in interlacing bundles. The nuclei were plump, oval, and sometimes indented. There was a distinct nucleolus, and often a central nuclear fold running in the long axis of the nucleus. Mitotic figures were scanty. The cytoplasm was finely granular, and the cytoplasmic membrane poorly defined. Numerous thin-walled vessels were present throughout, and sometimes the cells were orientated at right angles to them. In a few areas only, melanin was found in the cytoplasm, generally as a cluster of coarse granules. Although the main pattern of the cells was of the spindle cell variety, some areas were found where the cells were polygonal, with clear cytoplasm, and arranged into alveoli by delicate connective tissue strands. No nerve fibres were found within the tumour.

The capsule consisted of uncompressed fibro-fatty tissue containing muscle fibres, and was invaded by small islands of tumour cells.

This is obviously a melanoma. Histology will not reveal whether it is primary or secondary at this site.

Progress.-The operation was followed by marked oedema and ptosis of the upper lid, but on February 18 it was possible to open the eye, and the exophthalmos was decreasing. Although movement upward and inward was still limited, the visual acuity with $+3 \mathrm{D}$ sph. was $6 / 9$, add $+2 \cdot 25=$ N.6. The visual field had also begun to improve (Fig. 6, overleaf). Six months later, on June 15, the patient could read N.5 print and the exophthalmos and papilloedema had disappeared. No campimetric study was made on this occasion. 
FIG. 6.-Scotomata 5/2000$15 / 2000$ red field, left eye, on February 16, 1956, 10 days after operation.

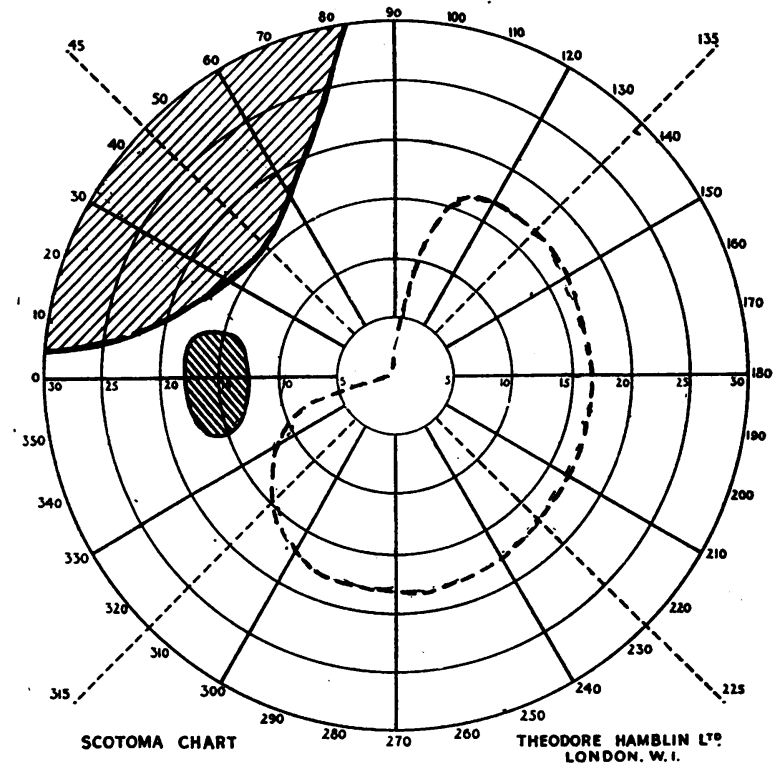

Discussion

Slides of the orbital growth were submitted to Dr. Norman Ashton at the Institute of Ophthalmology. Fig. 7 shows the original melanoma of the choroid.

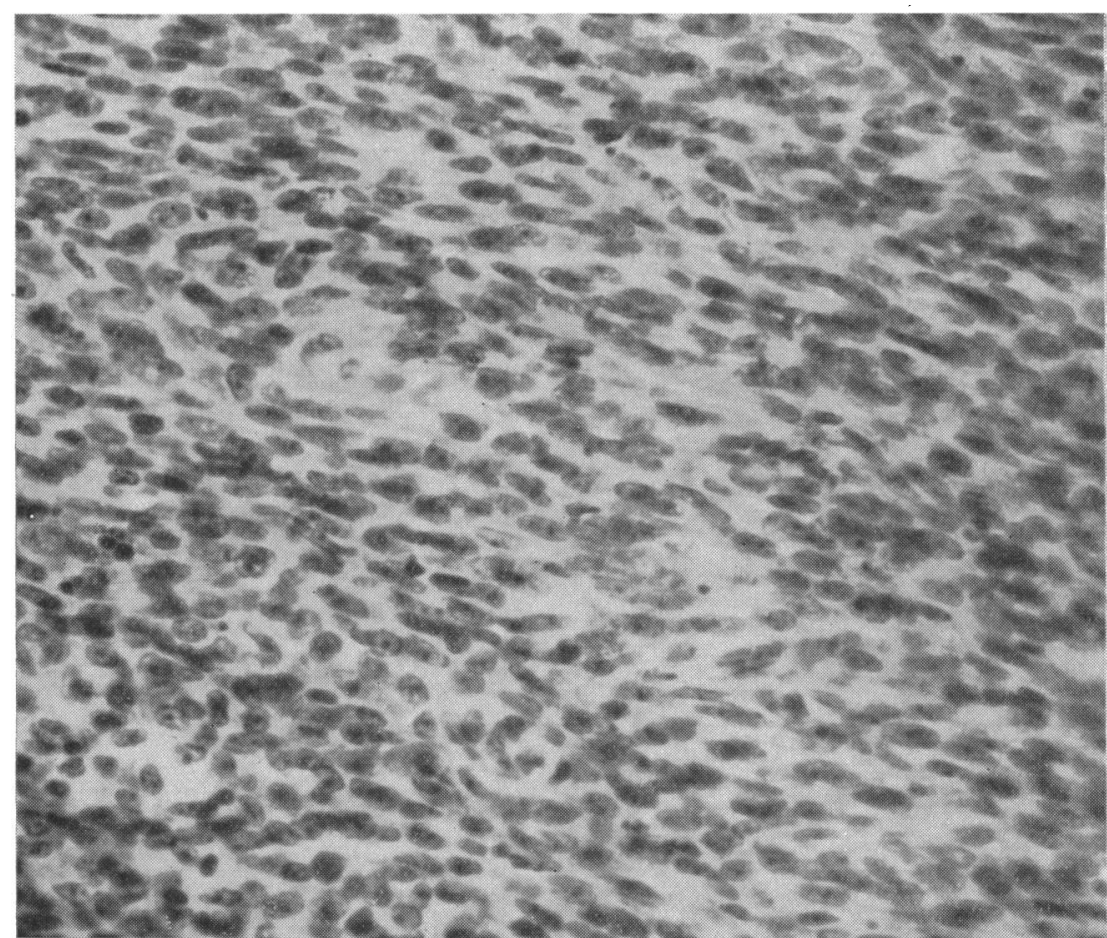

Fig. 7.-Section of melanotic sarcoma of right eye (courtesy of Dr. N. Ashton). $\quad \times 500$. 
Fig. 8 shows the orbital growth.

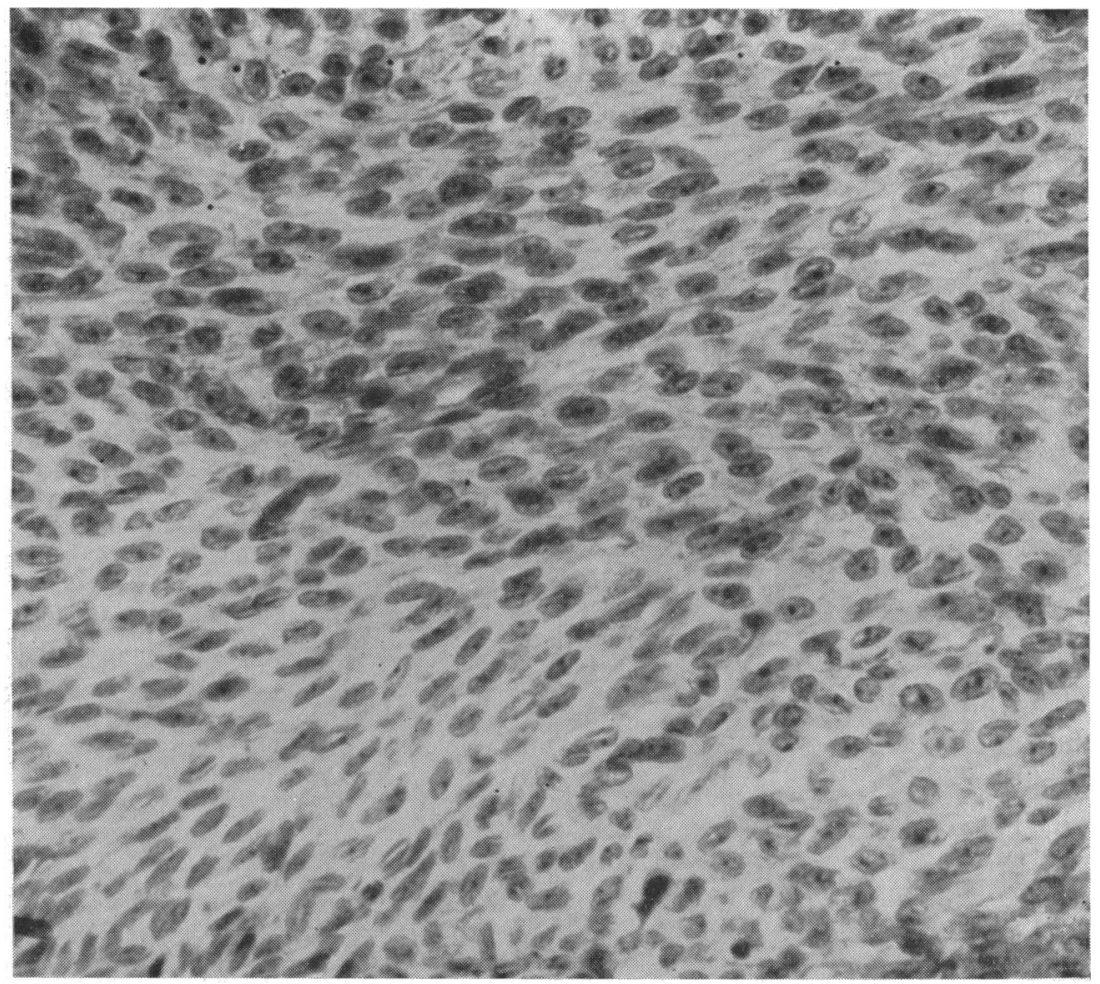

FIG. 8. - Section of orbital tumour (courtesy of Dr. N. Ashton). $\times 500$.

We are most grateful to Dr. Ashton for the following comment:

I have examined the slides of the original intra-ocular growth together with the slides of the orbital tumour. There is no doubt that the histology is identical, both showing a malignant spindle-celled melanoma, so that it would appear fairly certain that the tumour of the left orbit was a delayed metastasis from the right eye.

\section{Summary}

A rare case of metastasis of the contralateral orbit from a choroidal melanotic sarcoma is described. Transfrontal exploration was indicated by a hemianopic scotoma (which suggested a tumour was pressing on the inner side of the nerve) and localization by Diodone. Subtotal removal of the tumour had restored vision to normal 6 months later, but in September, 1956, the family doctor reported that the liver was enlarged and that the exophthalmos had recurred.

\section{REFERENCES}

Cowie, J. W., and Groves, J. S. (1955). British Journal of Ophthalmology, 39, 283. Philps, S., and Ashton, N. (1949). Ibid., 33, 732, 734. 